\title{
Design, synthesis and antitubercular evaluation of benzothiazinones containing a piperidine moiety
}

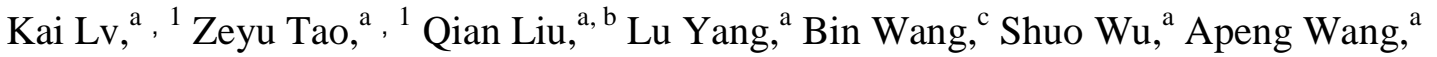 \\ Menghao Huang, ${ }^{\mathrm{d}}$ Mingliang Liu, ${ }^{\mathrm{a},}{ }^{*} \mathrm{Yu} \mathrm{Lu}^{\mathrm{c}},{ }^{*}$ \\ ${ }^{a}$ Institute of Medicinal Biotechnology, Chinese Academy of Medical Sciences and Peking Union Medical College, \\ Beijing 100050, China \\ ${ }^{\mathrm{b}}$ Institute of Molecular Design and Synthesis, School of Pharmaceutical Science \& Technology, Tianjin University, 92 \\ Weijin Road, Nankai District, Tianjin, 300072, P. R. China. \\ c Beijing Key Laboratory of Drug Resistance Tuberculosis Research, Department of Pharmacology, Beijing \\ Tuberculosis and Thoracic Tumor Research Institute, Beijing Chest Hospital, Capital Medical University, Beijing \\ 101149, China \\ ${ }^{\mathrm{d}}$ Division of Gastroenterology and Hepatology, Department of Medicine, Indiana University School of Medicine, \\ Indiana 46202, USA \\ ${ }^{1}$ These authors contributed equally to the work. \\ *Corresponding author: 1mllyx@126.com, 86-010-63030965 (M.L. Liu); luyu4876@hotmail.com, 86-010-89509357 \\ (Y. Lu)
}

\section{ABSTRACT}

We herein report the design and synthesis of benzothiazinones containing a piperidine moiety as new antitubercular agents based on the structure feature of IMB-ZR-1 discovered in our lab. Some of them were found to have good in vitro activity (MIC $<1 \mu \mathrm{g} / \mathrm{mL}$ ) against drug-susceptible Mycobacterium tuberculosis H37RV strain. After two set of modifications, compound $2 \mathbf{i}$ were found to display comparable in vitro anti-TB activity (MIC $<0.016 \mu \mathrm{g} / \mathrm{mL}$ ) to PBTZ169 against drug-sensitive and resistant mycobacterium tuberculosis strains. Compound 2i also showed acceptable PK profiles. Studies to determine PK profiles in lung and in vivo efficacy of $\mathbf{2 i}$ are currently under way.

KEYWORDS: benzothiazionones, PBTZ169, antimycobacterial activity, synthesis

\section{Introduction}

Tuberculosis (TB) is caused mainly by mycobacterium tuberculosis (MTB), has existed for millennia and remains a major global health problem.[1] The World Health Organization (WHO) estimated that approximately 10.4 million people were infected and 1.3 million died from TB worldwide in 2016.[2] The current guidelines for treatment of drug-susceptible TB infection recommends a combination of four front-line drugs rifampin, isoniazid, pyrazinamide and ethambutol for 6-9 months, often leading to significant side effects and poor patient compliance. In addition, the spread of multidrug-resistant (MDR) TB and extensively drug-resistant (XDR) TB has rendered these front-line drugs ineffective. [3] Therefore, there is an urgent need for discovery of new drugs with new mechanisms of action. Although Bedaquiline (inhibition of mitochondrial ATP synthase) and Delamanid (inhibition mycolic acid biosynthesis) were approved for the treatment of MDR-TB, over a huge gap of over 40 years, [4-5] both of them have pronounced issues, including hERG toxicity concerns, as well as multiple ADME issues due to their high lipophilicity. [6]

Benzothiazinones (BTZs), a novel class of TB agents were reported to have strong inhibitory potency against drug-sensitive TB, MDR-TB and XDR-TB strains targeting on decaprenyl phosphoryl- $\beta$-D-ribose 2 '-epimerase (DprE1). [7-12] The most advanced compound from this series (PBTZ169, Figure 1), developed by the Swiss Federal

This is the author's manuscript of the article published in final edited form as: 


\section{Graphic abstracts:}

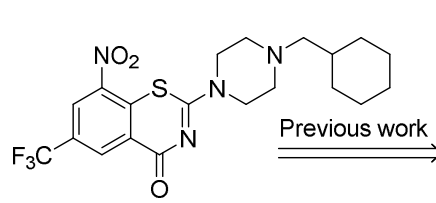

PBTZ169

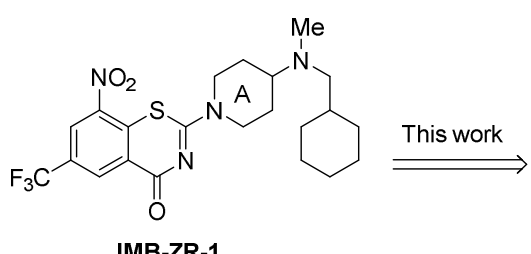

IMB-ZR-1<smiles>O=c1nc(N2CCC(CN3CCC(C(F)(F)F)CC3)CC2)sc2c([N+](=O)[O-])cc(C(F)(F)F)cc12</smiles>

Anti-TB activity: MIC $<0.016$ Anti-MDR-TB activity: MIC $<0.016$

New benzothiazinones bearing a piperidine moiety were designed and synthesized as antitubercular agents based on the structure feature of IMB-ZR-1 discovered in our lab. Compound $2 \mathbf{i}$ were found to display potent anti-TB activity (MIC $<0.016 \mu \mathrm{g} / \mathrm{mL}$ ) against drug-sensitive/resistant MTB strains. 
Institute of Technology and Innovative Medicines, has progressed into Phase II clinical trials in Russian Federation for the treatment of both drug-susceptible TB and MDR-TB.[2]

\section{$<$ INSERT FIGURE 1 HERE >}

The structure-activity relationship (SAR) and mechanistic studies of BTZs suggest that the $\mathrm{NO}_{2}$ group at position 8 and the sulfur atom at position 1 are critical for activity, that the $-\mathrm{CF}_{3}$ at position 6 plays an important role in maintaining activity. [7, 13-20] In our previous study, we focused on the modification at position 2, some of the resulting compounds were found to have improved activity and pharmacokinetic properties. [11, 12] Among them, IMB-ZR-1 with the piperidine ring displayed potent in vitro anti-TB activity against the tested MDR-TB strains (MIC $=0.016 \mu \mathrm{g} / \mathrm{mL}$ ). [12] Inspired by the above research results, we designed and synthesized two series of new BTZs 1-2 with piperidine moiety as ring A (Figure 1.). Shorten the linker between the two cycles of IMB-ZR-1 by removing the methylene group gave the series $\mathbf{1}$; shifting the nitrogen atom in the linker to ring B provided the series $\mathbf{2}$. In order to explore SAR of these BTZs, the R group on series $\mathbf{1}$ could be methyl or hydrogen; the W moiety of series $\mathbf{2}$ could be piperidine, pyrrolidine, azepane, 4-methylpiperazine, diethylamino, or substituted piperidine, et al. The antimycobacterial activity of all the target compounds were evaluated against drug-susceptible TB strain. The potent compound $2 \mathbf{i}$ was further evaluated for antimycobacterial activity against MDR-TB strains, and in vivo PK properties, aiming to identify alternative group at position 2 of BTZs and find more effective DprE1 inhibitors as anti-TB candidates.

\section{$<$ INSERT SCHEME 1 HERE>}

\section{Results and Discussion}

\subsection{Chemistry}

Detailed synthetic pathways to target compounds $\mathbf{1 a - b}$ and $\mathbf{2 a - k}$ are outlined in scheme 1. Reductive amination of ketone $\mathbf{3}$ and $\mathrm{N}$-methylcyclohexylamine or cyclohexylamine by $\mathrm{NaCNBH}_{3}$ in $\mathrm{MeOH}$ afforded compounds $\mathbf{4 a - b}$. Boc deprotection by TFA in DCM gave the intermediates $\mathbf{5 a - b}$. Coupling of $\mathbf{5} \mathbf{a}-\mathbf{b}$ and $\mathbf{6}$ which was prepared according literature procedure [8] furnished the BTZs 1a-b. Condensation of the acid 7 with amines in the presence of EDC and HOBt yielded compounds 8a-k. Removal of the Boc group followed by amide reduction with $\mathrm{LiAlH}_{4}$ formed intermediates 9a-k. Installation of $\mathbf{9 a - k}$ to $\mathbf{6}$ in the same manner as the preparation of 1a-b gave targets $\mathbf{2 a - k}$.

\subsection{Anti-TB activity}

The two series 1a-b and 2a-e bearing different kinds of amines to ensure the structure diversity were first synthesized (Table 1). They were preliminarily screened for in vitro activity against MTB H37Rv ATCC27395 strain, using the Microplate Alamar Blue Assay (MABA). [21] The minimum inhibitory concentration (MIC) is defined as the lowest concentration effecting a reduction in fluorescence of $>90 \%$ relative to the mean of replicate bacterium-only controls. The MIC values of the compounds along with PBTZ169, isoniazid (INH), and rifampicin (RFP) for comparison were obtained from three independent experiments and presented in $\mu \mathrm{g} / \mathrm{mL}$ in Table 1 .

\section{$<$ INSERT TABLE 1 HERE>}

The data revealed that these two BTZ derivatives 1a-b and 2a-e shown considerable anti-TB activity against this strain (MIC $<10 \mu \mathrm{g} / \mathrm{mL}$ ), although they were less active than PBTZ169, INH and RFP. Among them, compounds 1a-b and 2a displayed better activity (MIC $<1 \mu \mathrm{g} / \mathrm{mL}$ ) than other BTZs $\mathbf{2 b - e . ~ R e m o v a l ~ o f ~ t h e ~ m e t h y l ~ g r o u p ~ o n ~ 1 a ~ d i d n ' t ~}$ influence the potency (1a vs 1b). Decrease or increase the ring size of the piperidine group on 2a resulted in a loss of anti-TB activity (2a $v s \mathbf{2 b} v s \mathbf{2 c}$ ). Replacement of the piperidine group with $N$-methyl piperazine or diethyl amine also leaded to a decreased potency (2a vs $\mathbf{2 d}$ vs 2e). Specifically, compound 2d shown more than 16-fold higher MIC value 
than that of 2a. Consequently, this set of modification suggested that the cyclohexylamino (1a-b) and piperidin-1-ylmethyl (2a) moiety were more suitable for the $\mathrm{Q}$ fragment (Table 1) than other moieties. Considering compound 2a displayed best activity ( $\mathrm{MIC}=0.481 \mu \mathrm{g} / \mathrm{mL}$ ) among these two series, we intend to make further optimization based on $\mathbf{2 a}$.

\section{<INSERT TABLE 2 HERE>}

Next, an additional set of substituted piperidine analogues as $\mathrm{W}$ moiety listed in Table 2 were designed and synthesized. Our results indicated that some of them (2g-i) exhibited good activity (MIC $<1 \mu \mathrm{g} / \mathrm{mL}$ ). Among them, compound $2 \mathbf{i}$ (MIC $<0.016 \mu \mathrm{g} / \mathrm{mL})$ is more active than $\mathrm{INH}(\mathrm{MIC}=0.037 \mu \mathrm{g} / \mathrm{mL})$ and RFP $(\mathrm{MIC}=0.084 \mu \mathrm{g} / \mathrm{mL}$ ), and comparable to PBTZ169 (MIC $<0.016 \mu \mathrm{g} / \mathrm{mL}$ ). Exploration of SAR was first conducted by introduction of methyl group to ortho-, meta-, and para-positions of piperazine ring. Introduction of methyl group to ortho-position provided decreased potency (2a $v s \mathbf{2 f}$ ), whereas the presence of meta- or para-methyl group afforded a slightly increased or maintained anti-TB activity ( $\mathbf{2 a} v s \mathbf{2 g}$ and $\mathbf{2 h}$ ). The installation of hydroxy or ketal groups at the para-position was detrimental to the potency ( $\mathbf{2} \mathbf{a} v s \mathbf{2} \mathbf{j}$ and $\mathbf{2} \mathbf{k}$ ). Surprisingly, the introduction of trifluoro group at the para position caused a dramatical improvement in potency (2a $v s \mathbf{2 i})$.

Inspired by the strong anti-TB potency against drug sensitive strain, compound $\mathbf{2 i}$ was further evaluated against two clinical isolated MDR-TB strains (16995 and 16833) resistant to both INH and RFP (MIC > $40 \mu \mathrm{g} / \mathrm{mL}$ ). As shown in table 3, compound $2 \mathbf{i}$ (MIC $<0.016 \mu \mathrm{g} / \mathrm{mL}$ ) displayed comparable anti-MDR-TB activity to PBTZ169 (MIC < 0.016 $\mu \mathrm{g} / \mathrm{mL}$ ), suggesting its promising potential for both drug-sensitive and resistant TB strains.

\section{<INSERT TABLE 3 HERE>}

\subsection{Pharmacokinetics}

We subsequently investigated the in vivo $\mathrm{PK}$ profiles of compound $\mathbf{2 i}$ in mice after a single oral administration of 50 $\mathrm{mg} / \mathrm{kg}$. As shown in Table 4, compound $2 \mathbf{i}$ showed acceptable PK properties although the AUC of $\mathbf{2} \mathbf{i}(2489 \mathrm{~h} \cdot \mathrm{ng} / \mathrm{mL})$ is less than half of PBTZ169 $(5478 \mathrm{~h} \cdot \mathrm{ng} / \mathrm{mL})$. The Tmax of $2 \mathbf{i}(0.25 \mathrm{~h})$ is shorter than PBTZ169 $(0.83 \mathrm{~h})$, indicating that 2i was absorbed faster than PBTZ169. The Cmax is comparable to PBTZ169. We speculated that the lower AUC of 2i probably due to extensive tissue distribution or excretion. Currently, the PK profiles of $\mathbf{2} \mathbf{i}$ in lung is under testing, which will give the lung distribution properties of $\mathbf{2 i}$.

\section{〈INSERT TABLE 4 HERE>}

\section{Conclusion}

In summary, two series of BTZ derivatives with piperidine ring were designed as new anti-TB agents through modifications of IMB-ZR-1. Most of them exhibited considerable in vitro inhibitory (MIC $<10 \mu \mathrm{g} / \mathrm{mL}$ ) activity against drug sensitive strain. Compound $\mathbf{2 i}$ with 4-trifuoromethyl piperidine group as the $\mathrm{W}$ moiety of series $\mathbf{2}$ displayed comparable in vitro anti-TB activity to PBTZ169 against drug-sensitive and resistant TB strains. Compound 2i also showed acceptable PK profiles, although its AUC is less than half of PBTZ169. Studies to determine further PK profiles in lung and in vivo efficacy of $\mathbf{2 i}$ are currently under way.

\section{Experimental protocols}

\subsection{Chemistry}

Melting points were determined in open capillaries and are uncorrected. ${ }^{1} \mathrm{H}$ NMR spectra were determined on a Varian Mercury-400 spectrometer in DMSO- $d_{6}$ or $\mathrm{CDCl}_{3}$ using tetramethylsilane as an internal standard. Electrospray ionization (ESI) mass spectra was obtained on an MDSSCIEX Q-Tap mass spectrometer. The reagents were all of analytical grade or chemically pure. TLC was performed on silica gel plates (Merck, ART5554 60F254).

4.2. Synthesis

4.2.1. General procedure for the preparation of compound $\mathbf{4 a - b}$ 
To a stirred solution of tert-butyl 4-oxopiperidine-1-carboxylate (200 mg, $0.67 \mathrm{mmol})$ in anhydrous $\mathrm{MeOH}(5 \mathrm{~mL})$ was added $N$-methylcyclohexanamine/cyclohexanamine, $\mathrm{NaCNBH}_{3}(166 \mathrm{mg}, 2.68 \mathrm{mmol})$ at room temperature. The mixture was adjusted to $\mathrm{pH} 6$ by acetic acid, and stirred for 3 hours. The mixture quenched by $\mathrm{H}_{2} \mathrm{O}(20 \mathrm{~mL})$, and extracted by DCM. The organic layer was dried over anhydrous $\mathrm{MgSO}_{4}$, filtered and concentrated. The residue was purified by chromatography on a silica gel column to yield compound $\mathbf{4 a - b}$.

4.2.1.1. tert-butyl 4-(cyclohexyl(methyl)amino)piperidine-1-carboxylate 4a

Following the general procedure, employing $N$-methylcyclohexanamine yielded compound $\mathbf{4 a}$ as a colorless oil in $40 \%$ yield, ${ }^{1} \mathrm{HNMR}\left(500 \mathrm{MHz}, \mathrm{CDCl}_{3}\right) \delta 4.30$ (2H, brs), 3.34 (1H, brs), 3.17 (1H, brs), 2.73 (2H, brs), 2.65 (3H, s), $2.20(2 \mathrm{H}, \mathrm{m}), 1.96(2 \mathrm{H}, \mathrm{m}), 1.73(2 \mathrm{H}, \mathrm{m}), 1.58(2 \mathrm{H}, \mathrm{brs}), 1.42(9 \mathrm{H}, \mathrm{s}), 1.25(7 \mathrm{H}, \mathrm{brs}) ; \mathrm{MS}-\mathrm{ESI}(\mathrm{m} / \mathrm{z}): 319(\mathrm{M}+\mathrm{Na})^{+}$. 4.2.1.2. tert-butyl 4-(cyclohexylamino)piperidine-1-carboxylate $\mathbf{4 b}$

Following the general procedure, employing cyclohexanamine yielded compound $\mathbf{4 b}$ as a colorless oil in $50 \%$ yield, MS-ESI (m/z): $283(\mathrm{M}+\mathrm{H})^{+}$.

4.2.2. General procedure for the procedure of compound $\mathbf{5 a - b}$

To a stirred solution of $\mathbf{4 a - b}(0.25 \mathrm{mmol})$ in DCM $(5 \mathrm{~mL})$ was added TFA, stirred for 1 hour, and concentrated. The residue was diluted by DCM, concentrated again for three times to gave the crude $\mathbf{5} \mathbf{a}-\mathbf{b}$ which was used directly for the next step.

4.2.3 General procedure for the preparation of compound 8a-k

To a stirred solution of compound $7(200 \mathrm{mg}, 0.87 \mathrm{mmol})$ in DCM $(10 \mathrm{~mL})$ was added amines $(1.0 \mathrm{mmol})$, EDC (186 mg, $1.2 \mathrm{mmol})$, and HOBt $(153 \mathrm{mg}, 1.2 \mathrm{mmol})$ at room temperature. The mixture was stirred for $3 \mathrm{hrs}$, quenched by $\mathrm{H}_{2} \mathrm{O}(15 \mathrm{~mL})$, and extracted by DCM $(15 \mathrm{~mL} \times 3)$. The organic layer was dried over anhydrous MgSO4, filtered, and concentrated. The residue was purified by chromatography on a silica gel column to afford compound 8a-1 as a colorless oil.

4.2.3.1. tert-butyl 4-(piperidine-1-carbonyl)piperidine-1-carboxylate 8a

Following the general procedure, employing piperidine as the amine yielded compound $\mathbf{8 a}$ as a colorless oil in $85 \%$ yield, ${ }^{1} \mathrm{H}$ NMR (500 MHz, $\mathrm{CDCl}_{3}$ ) $\delta 4.14$ (brs, 2H), 3.55 (brs,2H), 3.43 (brs, 2H), 2.76 (brs, 2H), 2.62 (m, 1H), 1.66 (m, $6 \mathrm{H}), 1.55$ (brs, 4H), 1.45 (s, 9H); MS-ESI (m/z): $297(\mathrm{M}+\mathrm{H})^{+}$.

4.2.3.2. tert-butyl 4-(pyrrolidine-1-carbonyl)piperidine-1-carboxylate $\mathbf{8 b}$

Following the general procedure, employing piperidine as the amine yielded compound $\mathbf{8 b}$ as a colorless oil in $90 \%$ yield, which was used directly for the next step.

4.2.3.3. tert-butyl 4-(azepane-1-carbonyl)piperidine-1-carboxylate $\mathbf{8 c}$

Following the general procedure, employing azepane as the amine yielded compound $\mathbf{8 c}$ as a colorless oil in $94 \%$ yield, ${ }^{1} \mathrm{H}$ NMR (500 MHz, $\mathrm{CDCl}_{3}$ ) $\delta 4.15(\mathrm{brs}, 2 \mathrm{H}), 3.51$ (brs, $\left.2 \mathrm{H}\right), 3.47(\mathrm{t}, \mathrm{J}=5.9 \mathrm{~Hz}, 2 \mathrm{H}), 2.75(\mathrm{brs}, 2 \mathrm{H}), 2.60(\mathrm{~m}, 1 \mathrm{H})$, 2.01(m, 2H), 1.73(m, 6H), 1.57(brs, 4H), 1.46(s, 9H); MS-ESI (m/z): $333(\mathrm{M}+\mathrm{Na})^{+}$.

4.2.3.4. tert-butyl 4-(4-methylpiperazine-1-carbonyl)piperidine-1-carboxylate $\mathbf{8 d}$

Following the general procedure, employing azepane as the amine yielded compound $\mathbf{8 d}$ as a colorless oil in $75 \%$ yield, ${ }^{1} \mathrm{H}$ NMR (500 MHz, $\mathrm{CDCl}_{3}$ ) $\delta 4.14$ (brs, 2H), 3.65 (brs, 2H), 3.53 (brs, 2H), 2.75 (brs, 2H), $2.61(\mathrm{~m}, 1 \mathrm{H}), 2.41(\mathrm{~d}$, $\mathrm{J}=13.4 \mathrm{~Hz}, 4 \mathrm{H}), 2.32(\mathrm{~s}, 3 \mathrm{H}), 1.67(\mathrm{~m}, 4 \mathrm{H}), 1.45(\mathrm{~s}, 9 \mathrm{H}) ; \mathrm{MS}-\mathrm{ESI}(\mathrm{m} / \mathrm{z}): 312(\mathrm{M}+\mathrm{H})^{+}, 334(\mathrm{M}+\mathrm{Na})^{+}$.

4.2.3.5. tert-butyl 4-(diethylcarbamoyl)piperidine-1-carboxylate $\mathbf{8 e}$

Following the general procedure, employing diethylamine as the amine yielded compound $\mathbf{8 e}$ as a colorless oil in 80\% yield, ${ }^{1} \mathrm{H}$ NMR $\left(500 \mathrm{MHz}, \mathrm{CDCl}_{3}\right.$ ) $\delta 4.15$ (brs, 2H), 3.34 (q, J = 9.3 Hz, 4H), 2.74 (brs, 2H), 2.54 (t, J = 11.0 HZ, 1H), $1.75(\mathrm{q}, \mathrm{J}=10.1 \mathrm{~Hz}, 2 \mathrm{H}), 1.63(\mathrm{~m}, 2 \mathrm{H}), 1.45(\mathrm{~s}, 9 \mathrm{H}), 1.20(\mathrm{t}, \mathrm{J}=6.9 \mathrm{HZ}, 3 \mathrm{H}), 1.09$ (t, J = 6.9 HZ, 3H); MS-ESI $(\mathrm{m} / \mathrm{z}):(\mathrm{M}+\mathrm{H})^{+}$; MS-ESI $(\mathrm{m} / \mathrm{z}): 285(\mathrm{M}+\mathrm{H})^{+}$. 
4.2.3.6. tert-butyl 4-(2-methylpiperidine-1-carbonyl)piperidine-1-carboxylate $\mathbf{8 f}$

Following the general procedure, employing 2-methylpiperidine as the amine yielded compound $\mathbf{8 f}$ as a colorless oil in $87 \%$ yield, ${ }^{1} \mathrm{H}$ NMR $\left(500 \mathrm{MHz}, \mathrm{CDCl}_{3}\right) \delta 4.92$ (brs, $\left.1 \mathrm{H}\right), 4.49(\mathrm{~m}, 1 \mathrm{H}), 4.15$ (brs, 3H), 3.66 (m, 1H), 3.13 (m, 1H), 2.76 (brs, 2H), 2.59 (brs, 1H), 1.63 (m, 8H), 1.45 (s, 9H), 1.27 (brs, 3H); MS-ESI (m/z): $333(\mathrm{M}+\mathrm{Na})^{+}$.

4.2.3.7. tert-butyl 4-(3-methylpiperidine-1-carbonyl)piperidine-1-carboxylate $8 \mathrm{~g}$

Following the general procedure, employing 3-methylpiperidine as the amine yielded compound $\mathbf{8 g}$ as a colorless oil in $87 \%$ yield, ${ }^{1} \mathrm{H}$ NMR $\left(500 \mathrm{MHz}, \mathrm{CDCl}_{3}\right) \delta 4.43(\mathrm{~m}, 1 \mathrm{H}), 4.14(\mathrm{brs}, 2 \mathrm{H}), 3.80(\mathrm{~d}, \mathrm{~J}=11.8 \mathrm{~Hz}, 1 \mathrm{H}), 3.71(\mathrm{~d}, \mathrm{~J}=13.4$ $\mathrm{Hz}, 1 \mathrm{H}), 2.98(\mathrm{~m}, 1 \mathrm{H}), 2.76$ (brs, 2H), $2.63(\mathrm{~m}, 1 \mathrm{H}), 2.24$ (t, J = 11.2 Hz, 1H), $1.83(\mathrm{~d}, \mathrm{~J}=12.5 \mathrm{~Hz}, 1 \mathrm{H}), 1.68(\mathrm{~m}, 5 \mathrm{H})$, 1.45 (brs, 9H), $1.25(\mathrm{~m}, 1 \mathrm{H}), 1.15(\mathrm{~m}, 1 \mathrm{H}), 0.92(\mathrm{~d}, \mathrm{~J}=10.7 \mathrm{~Hz}, 3 \mathrm{H})$; MS-ESI $(\mathrm{m} / \mathrm{z}): 333(\mathrm{M}+\mathrm{Na})^{+}$.

4.2.3.8. tert-butyl 4-(4-methylpiperidine-1-carbonyl)piperidine-1-carboxylate $\mathbf{8 h}$

Following the general procedure, employing 4-methylpiperidine as the amine yielded compound $\mathbf{8 h}$ as a colorless oil in $87 \%$ yield, ${ }^{1} \mathrm{H}$ NMR (500 MHz, $\left.\mathrm{CDCl}_{3}\right) \delta 4.57$ (brs, $\left.1 \mathrm{H}\right), 4.14$ (brs, $\left.2 \mathrm{H}\right), 3.85$ (brs, $\left.1 \mathrm{H}\right), 3.01$ (brs, $\left.1 \mathrm{H}\right), 2.76(\mathrm{~m}$, 2H), $2.62(\mathrm{~m}, 1 \mathrm{H}), 2.54(\mathrm{~m}, 1 \mathrm{H}), 1.66(\mathrm{~m}, 7 \mathrm{H}), 1.45(\mathrm{~s}, 9 \mathrm{H}), 1.08(\mathrm{~m}, 2 \mathrm{H}), 0.95(\mathrm{~d}, \mathrm{~J}=6.5 \mathrm{~Hz}, 2 \mathrm{H})$.

4.2.3.9. tert-butyl 4-(4-(trifluoromethyl)piperidine-1-carbonyl)piperidine-1-carboxylate $\mathbf{8 i}$

Following the general procedure, employing 4-trifluoromethylpiperidine as the amine yielded compound $\mathbf{8} \mathbf{i}$ as a colorless oil in 68\% yield, ${ }^{1} \mathrm{H}$ NMR $\left(500 \mathrm{MHz}, \mathrm{CDCl}_{3}\right) \delta 4.75(\mathrm{~d}, \mathrm{~J}=11.2 \mathrm{~Hz}, 1 \mathrm{H}), 4.14(\mathrm{brs}, 2 \mathrm{H}), 4.01(\mathrm{~d}, \mathrm{~J}=11.4 \mathrm{~Hz}$, $1 \mathrm{H}), 3.05(\mathrm{~m}, 1 \mathrm{H}), 2.77$ (brs, 2H), $2.61(\mathrm{~m}, 1 \mathrm{H}), 2.53(\mathrm{~m}, 1 \mathrm{H}), 2.28(\mathrm{~m}, 1 \mathrm{H}), 1.94(\mathrm{~m}, 2 \mathrm{H}), 1.72(\mathrm{~m}, 2 \mathrm{H}), 1.66(\mathrm{brs}, 2 \mathrm{H})$, 1.45 (brs, 11H); MS-ESI (m/z): $365(\mathrm{M}+\mathrm{H})^{+}$.

4.2.3.10. tert-butyl 4-(4-hydroxypiperidine-1-carbonyl)piperidine-1-carboxylate $\mathbf{8 j}$

Following the general procedure, employing 4- hydroxypiperidine as the amine yielded compound $\mathbf{8 j}$ as a colorless oil in 68\% yield, ${ }^{1} \mathrm{H}$ NMR $\left(500 \mathrm{MHz}, \mathrm{CDCl}_{3}\right) \delta 4.08(\mathrm{~m}, 2 \mathrm{H}), 3.95$ (brs, 2H), 3.76 (brs, $\left.1 \mathrm{H}\right), 3.23(\mathrm{~m}, 2 \mathrm{H})$, 2.76 (brs, 2H), 2.63 (brs, 1H), 2.07 (m, 2H), 1.89 (brs, 2H), 1.69 (brs, 2H), 1.50 (m, 2H), 1.45 (brs, 9H); MS-ESI (m/z): $335(\mathrm{M}+\mathrm{Na})^{+}$.

4.2.3.11. tert-butyl 4-(1,4-dioxa-8-azaspiro[4.5]decane-8-carbonyl)piperidine-1-carboxylate $\mathbf{8 k}$

Following the general procedure, employing 4- hydroxypiperidine as the amine yielded compound $\mathbf{8 k}$ as a colorless oil in 75\% yield, ${ }^{1} \mathrm{H}$ NMR (500 MHz, $\left.\mathrm{CDCl}_{3}\right) \delta 4.14(\mathrm{~d}, \mathrm{~J}=11.6 \mathrm{~Hz}, 2 \mathrm{H}), 3.98(\mathrm{~s}, 4 \mathrm{H}), 3.68(\mathrm{brs}, 2 \mathrm{H}), 3.56$ (brs, 2H), $2.76(\mathrm{t}, \mathrm{J}=12.2 \mathrm{~Hz}, 2 \mathrm{H}), 2.64(\mathrm{~m}, 1 \mathrm{H}), 1.69(\mathrm{~m}, 8 \mathrm{H}), 1.45(\mathrm{~s}, 9 \mathrm{H}) ;$ MS-ESI $(\mathrm{m} / \mathrm{z}): 355(\mathrm{M}+\mathrm{H})^{+}$.

4.2.4. General procedure for the preparation of $\mathbf{9 a - k}$

To a stirred solution of $\mathbf{8 a - k}(0.25 \mathrm{mmol})$ in DCM $(5 \mathrm{~mL})$ was added TFA, and stirred for 1 hour. The mixture was concentrated, and diluted by DCM, concentrated again for three times to fully remove the TFA. The residue was dissolved in THF. To the mixture was added $\mathrm{LiAlH}_{4}\left(0.5 \mathrm{~mL}, 1 \mathrm{M}\right.$ solution in THF) at $0{ }^{\circ} \mathrm{C}$, and stirred for $30 \mathrm{mins}$. The mixture was slowly quenched by $\mathrm{MeOH}(0.5 \mathrm{~mL})$, and $\mathrm{NaOH}$ solution $(0.2 \mathrm{~mL}, 1 \mathrm{M})$. The mixture was filtered through celite, washed by $\mathrm{MeOH}$, and concentrated. The residue was concentrated and purified by chromatography on a silica gel column ( $\mathrm{DCM}: \mathrm{MeOH}: \mathrm{NH}_{3} \cdot \mathrm{H}_{2} \mathrm{O}=5: 3: 1$ ) to afford crude 9a-k as brown oils, which were used directly for the next step.

4.2.5. General procedure for the preparation of $\mathbf{1 a - b}$ and $\mathbf{2 a - k}$

To a stirred solution of $\mathbf{5 a - b}$ or $\mathbf{9 a - k}(0.1 \mathrm{mmol})$ in anhydrous $\mathrm{MeOH}(5 \mathrm{~mL})$ was added compound $\mathbf{6}(32 \mathrm{mg}, 0.1$ $\mathrm{mmol})$ and $\mathrm{Et}_{3} \mathrm{~N}(28 \mu \mathrm{L}, 0.2 \mathrm{mmol})$ at room temperature under argon. The mixture was heated to $40{ }^{\circ} \mathrm{C}$, stirred for 3 hours, and concentrated. The mixture was diluted by DCM $(15 \mathrm{~mL})$ and washed by $\mathrm{H}_{2} \mathrm{O}$. The organic layer was dried over anhydrous $\mathrm{MgSO}_{4}$, filtered, and concentrated. The residue was purified by chromatography on a silica gel column 
(DCM : MeOH : $\mathrm{NH}_{3} \cdot \mathrm{H}_{2} \mathrm{O}=10: 1: 0.01$ ) or prepare TLC (DCM : MeOH $\left.: \mathrm{NH}_{3} \cdot \mathrm{H}_{2} \mathrm{O}=10: 1: 0.01\right)$ to give BTZs 1a-b or 2 a-k.

4.2.5.1. 2-(4-(cyclohexyl(methyl)amino)piperidin-1-yl)-8-nitro-6-(trifluoromethyl)-4H-benzo[e][1,3]thiazin-4-one 1a

Following the general procedure, employing 5a yielded compound 1a as yellow solid in $40 \%$ yield, mp: $146-148{ }^{\circ} \mathrm{C}$; HPLC purity, $98.1 \%$; ${ }^{1} \mathrm{H}$ NMR $\left(500 \mathrm{MHz}, \mathrm{CDCl}_{3}\right) \delta 9.09(1 \mathrm{H}, \mathrm{s}), 8.75(1 \mathrm{H}, \mathrm{s}), 5.24(1 \mathrm{H}, \mathrm{brs}), 4.43$ (1H, brs), 3.18 (2H,brs), 3.05 (1H, brs), 2.68 (1H,brs), 2.35 (3H, s), 2.05 (2H, brs), 1.83 (4H, brs), 1.65 (2H, d, J = $11.7 \mathrm{~Hz}), 1.37$ (2H, $\mathrm{m}), 1.26(2 \mathrm{H}, \mathrm{m}), 1.12(2 \mathrm{H}, \mathrm{m})$; ESI-MS $(\mathrm{m} / \mathrm{z}): 471(\mathrm{M}+\mathrm{H})^{+}$.

4.2.5.2. 2-(4-(cyclohexylamino)piperidin-1-yl)-8-nitro-6-(trifluoromethyl)-4H-benzo[e][1,3]thiazin-4-one 1b

Following the general procedure, employing $\mathbf{5 b}$ yielded compound $\mathbf{1 b}$ as yellow solid in $40 \%$ yield, mp: $171-173{ }^{\circ} \mathrm{C}$; HPLC purity, 96.3\%; ${ }^{1} \mathrm{H}$ NMR $\left(500 \mathrm{MHz}, \mathrm{CDCl}_{3}\right) \delta 9.09(1 \mathrm{H}, \mathrm{s}), 8.75(1 \mathrm{H}, \mathrm{s}), 4.99(1 \mathrm{H}, \mathrm{brs}), 4.34(1 \mathrm{H}, \mathrm{brs}), 3.48(1 \mathrm{H}$, s), 3.38 (2H, brs), $3.11(1 \mathrm{H}, \mathrm{brs}), 2.62(1 \mathrm{H}, \mathrm{brs}), 2.09(2 \mathrm{H}, \mathrm{brs}), 1.91(2 \mathrm{H}, \mathrm{d}, \mathrm{J}=9.65 \mathrm{~Hz}), 1.76(2 \mathrm{H}, \mathrm{d}, \mathrm{J}=12.3 \mathrm{~Hz})$, $1.63(2 \mathrm{H}, \mathrm{d}, \mathrm{J}=11.8 \mathrm{~Hz}), 1.53(2 \mathrm{H}, \mathrm{brs}), 1.26(2 \mathrm{H}, \mathrm{m}), 1.16(2 \mathrm{H}, \mathrm{m})$; ESI-MS $(\mathrm{m} / \mathrm{z}): 457(\mathrm{M}+\mathrm{H})^{+}$.

4.2.5.3. 8-nitro-2-(4-(piperidin-1-ylmethyl)piperidin-1-yl)-6-(trifluoromethyl)-4H-benzo[e][1,3]thiazin-4-one 2a

Following the general procedure, employing 9a yielded compound 2a as yellow solid in $40 \%$ yield, mp: $165-167{ }^{\circ} \mathrm{C}$; HPLC purity, 99.0\%; ${ }^{1} \mathrm{H}$ NMR $\left(500 \mathrm{MHz}, \mathrm{CDCl}_{3}\right) \delta(\mathrm{ppm}) 9.10(\mathrm{~s}, 1 \mathrm{H}), 8.74(\mathrm{~s}, 1 \mathrm{H}), 5.25$ (brs, $\left.1 \mathrm{H}\right), 4.37$ (brs, $\left.1 \mathrm{H}\right)$,

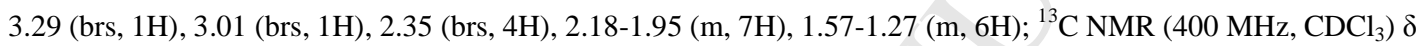
(ppm) 166.46, 161.62, 144.03, 134.28, 133.26 (q, J = 3.5 Hz), $129.61(\mathrm{q}, \mathrm{J}=35.4 \mathrm{~Hz}), 126.88,125.85$ (q, J = 3.6 Hz), $122.44(\mathrm{q}, \mathrm{J}=272.95 \mathrm{~Hz}), 64.36,55.20,46.94,33.63,30.84,25.63,24.16$; ESI-MS (m/z): $457(\mathrm{M}+\mathrm{H})^{+}$. 4.2.5.4. 8-nitro-2-(4-(pyrrolidin-1-ylmethyl)piperidin-1-yl)-6-(trifluoromethyl)-4H-benzo[e][1,3]thiazin-4-one 2b

Following the general procedure, employing $\mathbf{9 b}$ yielded compound $\mathbf{2 b}$ as yellow solid in $20 \%$ yield, mp:156-158 ${ }^{\circ} \mathrm{C}$; HPLC purity, 93.9\%; ${ }^{1} \mathrm{H}$ NMR $\left(500 \mathrm{MHz}, \mathrm{CDCl}_{3}\right) \delta(\mathrm{ppm}) 9.09$ (s, 1H), $8.74(\mathrm{~s}, 1 \mathrm{H}), 5.30-5.24(\mathrm{~m}, 1 \mathrm{H}), 4.38(\mathrm{brs}, 1 \mathrm{H})$, 3.91-3.05 (m, 2H), 2.71 (brs, 4H), 2.52 (brs, 3H), 2.21-2.00 (m, 4H), 1.87 (brs, $2 \mathrm{H}), 1.35-1.25(\mathrm{~m}, 2 \mathrm{H}),{ }^{13} \mathrm{C}$ NMR $(400$ $\left.\mathrm{MHz}, \mathrm{CDCl}_{3}\right) \delta(\mathrm{ppm}) 166.57,161.74,143.94,134.30,133.34$ (q, J = 3.4 Hz), $129.60(\mathrm{q}, \mathrm{J}=35.4 \mathrm{~Hz}), 126.73,126.00$ $(\mathrm{q}, \mathrm{J}=3.7 \mathrm{~Hz}), 122.42(\mathrm{q}, \mathrm{J}=273.5 \mathrm{~Hz}), 61.72,54.76,46.81,35.08,30.67,23.48$; ESI-MS (m/z): $443(\mathrm{M}+\mathrm{H})^{+}$. 4.2.5.5. 2-(4-(azepan-1-ylmethyl)piperidin-1-yl)-8-nitro-6-(trifluoromethyl)-4H-benzo[e][1,3]thiazin-4-one 2c

Following the general procedure, employing $\mathbf{9 c}$ yielded compound $\mathbf{2 c}$ as yellow solid in $28 \%$ yield, mp: $141-143{ }^{\circ} \mathrm{C}$; HPLC purity, 92.8\%; ${ }^{1} \mathrm{H}$ NMR $\left(500 \mathrm{MHz}, \mathrm{CDCl}_{3}\right) \delta(\mathrm{ppm}) 9.10(\mathrm{~s}, 1 \mathrm{H}), 8.74(\mathrm{~s}, 1 \mathrm{H}), 5.30-5.24(\mathrm{~m}, 1 \mathrm{H}), 4.38(\mathrm{brs}, 1 \mathrm{H})$, 3.29 (brs, $1 \mathrm{H}), 3.04$ (brs, $1 \mathrm{H}), 2.67$ (brs, $4 \mathrm{H}), 2.40$ (brs, $2 \mathrm{H}), 2.22-1.88(\mathrm{~m}, 5 \mathrm{H}), 1.62$ (d, J = 26.0 Hz, 6H), 1.28-1.25 (m, $2 \mathrm{H}) ;{ }^{13} \mathrm{C} \mathrm{NMR}\left(400 \mathrm{MHz}, \mathrm{CDCl}_{3}\right) \delta(\mathrm{ppm}) 166.61,161.62,143.95,134.39,133.34(\mathrm{q}, \mathrm{J}=35.3 \mathrm{~Hz}), 126.78,125.97$ (q, $\mathrm{J}=3.6 \mathrm{~Hz}), 122.44(\mathrm{q}, \mathrm{J}=272.4 \mathrm{~Hz}), 63.29,55.98,47.16,34.81,30.75,27.83,27.17$; ESI-MS (m/z): $471.6(\mathrm{M}+\mathrm{H})^{+}$. 4.2.5.6. 2-(4-((4-methylpiperazin-1-yl)methyl)piperidin-1-yl)-8-nitro-6-(trifluoromethyl)-4H-benzo[e][1,3] thiazin-4-one 2d

Following the general procedure, employing $9 \mathrm{~d}$ yielded compound $2 \mathrm{~d}$ as yellow solid in $28 \%$ yield, mp: $143-145^{\circ} \mathrm{C}$; HPLC purity, 95.1\%; ${ }^{1} \mathrm{H}$ NMR (500 MHz, $\mathrm{CDCl}_{3}$ ) $\delta(\mathrm{ppm}) 9.09$ (s, 1H), 8.74 (s, 1H), 5.25 (brs, 1H), 4.37 (brs, 1H), 3.28-3.20 (m, 1H), 3.11-3.00 (m, 1H), 2.53 (brs, 8H), 2.37 (s,3H), 2.24 (d, J = 6.6Hz, 2H), 1.99-1.90 (m, 3H), 1.28-1.26 $(\mathrm{m}, 2 \mathrm{H}) ;{ }^{13} \mathrm{C}$ NMR $\left(400 \mathrm{MHz}, \mathrm{CDCl}_{3}\right) \delta(\mathrm{ppm}) 166.57,161.67,143.95,134.32,133.35$ (q, J = $\left.3.5 \mathrm{~Hz}\right), 129.59$ (q, J = $35.5 \mathrm{~Hz}), 126.76,125.98$ (q, J = 3.6 Hz), 122.42 (q, J = 271.6 Hz), 63.53, 54.90, 52.96, 46.96, 45.58, 33.60, 30.67; ESI-MS (m/z): $472.6(\mathrm{M}+\mathrm{H})^{+}$.

4.2.5.7. 2-(4-((diethylamino)methyl)piperidin-1-yl)-8-nitro-6-(trifluoromethyl)-4H-benzo[e][1,3]thiazin-4-one 2e

Following the general procedure, employing 9 e yielded compound $\mathbf{2 e}$ as yellow solid in 38\% yield, mp: 117-118 ${ }^{\circ} \mathrm{C}$; HPLC purity, 92.9\%; ${ }^{1} \mathrm{H}$ NMR $\left(500 \mathrm{MHz}, \mathrm{CDCl}_{3}\right) \delta(\mathrm{ppm}) 9.07$ (s, 1H), $8.72(\mathrm{~s}, 1 \mathrm{H}), 5.28($ brs, $1 \mathrm{H}), 4.36$ (brs, $1 \mathrm{H}$ ), 
3.28 (brs, 1H), 3.06-2.99 (m, 1H), 2.51 (q, J = 7.0 Hz, 4H), 2.27 (d, J = 6.5 Hz, 2H), 2.02-1.99 (m, 2H), 1.86-1.81 (m, $1 \mathrm{H}), 1.30-1.21(\mathrm{~m}, 2 \mathrm{H}), 0.99(\mathrm{t}, 6 \mathrm{H})$; ESI-MS $(\mathrm{m} / \mathrm{z}): 445(\mathrm{M}+\mathrm{H})^{+}$.

4.2.5.8.

2-(4-((2-methylpiperidin-1-yl)methyl)piperidin-1-yl)-8-nitro-6-(trifluoromethyl)-4H-benzo[e][1,3]

thiazin-4-one $\mathbf{2 f}$

Following the general procedure, employing $\mathbf{9 e}$ yielded compound $\mathbf{2 e}$ as yellow solid in 36\% yield, mp: 128-130 ${ }^{\circ} \mathrm{C}$; HPLC purity, $98.1 \% ;{ }^{1} \mathrm{H}$ NMR $\left(500 \mathrm{MHz}, \mathrm{CDCl}_{3}\right) \delta(\mathrm{ppm}) 9.09(\mathrm{~s}, 1 \mathrm{H}), 8.74(\mathrm{~s}, 1 \mathrm{H}), 5.29-5.25(\mathrm{~m}, 1 \mathrm{H}), 4.37$ (brs, $1 \mathrm{H}), 3.29$ (brs, 1H), 3.00 (brs, 1H), 2.84 (brs, 1H), 2.54 (brs, 1H), 2.62-1.89 (m, 4H), 1.63 (brs, $4 \mathrm{H}), 1.28-1.25$ (m, 6H), $1.02(\mathrm{~s}, 3 \mathrm{H}) ;{ }^{13} \mathrm{C}$ NMR $\left(400 \mathrm{MHz}, \mathrm{CDCl}_{3}\right) \delta(\mathrm{ppm}) 166.58,161.57,143.39,133.34(\mathrm{q}, \mathrm{J}=3.5 \mathrm{~Hz}), 129.54(\mathrm{q}, \mathrm{J}=35.2$ $\mathrm{Hz}), 126.79,125.96$ (q, J = 3.7 Hz), 122.44 (q, J = 272.9 Hz), 59.31, 56.88, 53.12, 47.19, 34.71, 30.90, 25.93, 23.57, 19.05, 14.15; ESI-MS (m/z): $471(\mathrm{M}+\mathrm{H})^{+}$.

4.2.5.9. 2-(4-((3-methylpiperidin-1-yl)methyl)piperidin-1-yl)-8-nitro-6-(trifluoromethyl)-4H-benzo[e][1,3] thiazin-4-one $2 \mathrm{~g}$

Following the general procedure, employing $\mathbf{9 g}$ yielded compound $\mathbf{2 g}$ as yellow solid in 36\% yield, mp: 127-128 ${ }^{\circ} \mathrm{C}$; HPLC purity, $98.1 \% ;{ }^{1} \mathrm{H}$ NMR $\left(500 \mathrm{MHz}, \mathrm{CDCl}_{3}\right) \delta(\mathrm{ppm}) 9.10(\mathrm{~s}, 1 \mathrm{H}), 8.74(\mathrm{~s}, 1 \mathrm{H}), 5.34-5.25(\mathrm{~m}, 1 \mathrm{H}), 4.37$ (brs, $1 \mathrm{H}), 3.30$ (brs, 1H), 3.02 (brs, 1H), 2.72 (brs, 2H), 2.16 (brs, 2H), 2.04-1.85 (m, 5H), 1.70-1.55 (m, 5H), 1.26 (brs, 2H), $0.86(\mathrm{~d}, \mathrm{~J}=5.7 \mathrm{~Hz}, 3 \mathrm{H}) ;{ }^{13} \mathrm{C} \mathrm{NMR}\left(400 \mathrm{MHz}, \mathrm{CDCl}_{3}\right) \delta(\mathrm{ppm}) 166.60,161.62,143.95,134.39,133.34(\mathrm{q}, \mathrm{J}=3.4 \mathrm{~Hz})$, 129.56(q, J = 35.5 Hz), 126.79, 125.96 (q, J = 3.7 Hz), 122.44 (q, J = 273.8 Hz), 64.36, 62.79, 54.78, 47.12, 33.81, 32.98, 31.09, 29.35, 25.51, 19.71; ESI-MS (m/z): $471(\mathrm{M}+\mathrm{H})^{+}$.

4.2.5.10. 2-(4-((4-methylpiperidin-1-yl)methyl)piperidin-1-yl)-8-nitro-6-(trifluoromethyl)-4H-benzo[e][1,3] thiazin-4-one $\mathbf{2 h}$

Following the general procedure, employing $\mathbf{9 h}$ yielded compound $\mathbf{2 h}$ as yellow solid in 36\% yield, mp: 130-131 ${ }^{\circ} \mathrm{C}$; HPLC purity, $97.6 \% ;{ }^{1} \mathrm{H}$ NMR $\left(500 \mathrm{MHz}, \mathrm{CDCl}_{3}\right) \delta(\mathrm{ppm}) 9.14(\mathrm{~s}, 1 \mathrm{H}), 8.79(\mathrm{~s}, 1 \mathrm{H}), 5.39-5.28(\mathrm{~m}, 1 \mathrm{H}), 4.41$ (brs, 1H), 3.33 (brs, 1H), 3.06 (brs, 1H), 2.84 (brs, 1H), 2.22 (d, J = 4.0 Hz, 2H), 1.96 (brs, 5H), 1.66-1.63 (m, 3H), 1.30 (brs, $4 \mathrm{H}), 0.97(\mathrm{~d}, \mathrm{~J}=6.3 \mathrm{~Hz}, 3 \mathrm{H}) ;{ }^{13} \mathrm{C}$ NMR $\left(400 \mathrm{MHz}, \mathrm{CDCl}_{3}\right) \delta(\mathrm{ppm}) 166.58,161.62,143.94,133.33(\mathrm{q}, \mathrm{J}=3.5 \mathrm{~Hz})$, 129.54 (q, J = 35.4 Hz), 126.79, 125.95(q, J = 3.6 Hz), 122.44(q, J = 273.2 Hz), 63.21, 54.65, 53.46, 47.06, 34.27, $33.84,30.78,21.87$; ESI-MS (m/z): $471(\mathrm{M}+\mathrm{H})^{+}$.

4.2.5.11. 8-nitro-6-(trifluoromethyl)-2-(4-((4-(trifluoromethyl)piperidin-1-yl)methyl)piperidin-1-yl)-4H-benzo[e][1,3] thiazin-4-one $2 \mathbf{i}$

Following the general procedure, employing $\mathbf{9 i}$ yielded compound $\mathbf{2 i}$ as yellow solid in $36 \%$ yield, $\mathrm{mp}$ : 158-159 ${ }^{\circ} \mathrm{C}$; HPLC purity, $98.4 \% ;{ }^{1} \mathrm{H}$ NMR $\left(500 \mathrm{MHz}, \mathrm{CDCl}_{3}\right) \delta(\mathrm{ppm}) 9.14$ (s, 1H),8.79 (s, 1H),5.30 (brs, 1H), 4.43 (brs, 1H), 3.33 (brs, 1H), 2.98 (brs, 4H), 2.26 (brs, 2H), 2.01 (brs, 3H), 1.87 (brs, 4H), 1.66 (brs, 2H), 1.32 (d, J = 7.2 Hz, $2 \mathrm{H}) ;{ }^{13} \mathrm{C}$ NMR (400 MHz, $\left.\mathrm{CDCl}_{3}\right) \delta(\mathrm{ppm}) 166.58,161.70,143.95,134.30,133.35$ (q, J = 3.5 Hz), $129.61(\mathrm{q}, \mathrm{J}=35.5$ $\mathrm{Hz}), 126.75,126.00$ (q, J = 3.6 Hz), 122.42 (q, J = 272.5 Hz),63.73, 53.08, 46.92, 40.33 (q, J = 28.4 Hz), 33.83, 30.69, 24.63; ESI-MS (m/z): $525.6(\mathrm{M}+\mathrm{H})^{+}$.

4.2.5.12. 2-(4-((4-hydroxypiperidin-1-yl)methyl)piperidin-1-yl)-8-nitro-6-(trifluoromethyl)-4H-benzo[e][1,3] thiazin-4-one $2 \mathbf{j}$

Following the general procedure, employing $\mathbf{9 j}$ yielded compound $\mathbf{2} \mathbf{j}$ as yellow solid in $36 \%$ yield, mp: $78-80{ }^{\circ} \mathrm{C}$; HPLC purity, 93.4\%; ${ }^{1} \mathrm{H}$ NMR (500 MHz, $\left.\mathrm{CDCl}_{3}\right) \delta(\mathrm{ppm})$ 9.09(s, 1H),8.7 5(s, 1H), 5.26 (brs, 1H), 4.39 (brs, 1H), 3.71-3.52 (m, 1H), 3.29-3.04 (m, 5H), 2.73 (brs, 2H), 2.33-2.20 (m, 4H), 2.00-1.90 (m, 3H), 1.56 (brs, 2H), 1.26 (brs, $2 \mathrm{H}) ;{ }^{13} \mathrm{C}$ NMR $\left(400 \mathrm{MHz}, \mathrm{CDCl}_{3}\right) \delta(\mathrm{ppm}) 166.60,161.71,143.95,134.32,133.36$ (q, J = 3.3 Hz), 129.60 (q, J = 34.9 $\mathrm{Hz}), 126.75,126.00$ (q, J = 3.3 Hz), 122.42 (q, J = 273.1 Hz), 63.51, 51.60, 46.98, 34.47, 33.91, 30.79, 29.72, 29.35, 27.24; ESI-MS (m/z): $473.6(\mathrm{M}+\mathrm{H})^{+}$. 
4.2.5.13. 2-(4-((1,4-dioxa-8-azaspiro[4.5]decan-8-yl)methyl)piperidin-1-yl)-8-nitro-6-(trifluoromethyl)-4H-benzo[e]

[1,3]thiazin-4-one $\mathbf{2 k}$

Following the general procedure, employing $9 k$ yielded compound $\mathbf{2 k}$ as yellow solid in $36 \%$ yield, mp: $78-80{ }^{\circ} \mathrm{C}$; ${ }^{1} \mathrm{H}$ NMR (500 MHz, CDCl $) \delta 9.09$ (s, 1H), 8.75 (s, 1H), 5.34-5.25 (m, 1H), 4.41 (brs, 1H), 3.96 (brs, 4H), 3.28 (brs, $1 \mathrm{H}), 3.05$ (brs, 1H), 2.59 (brs, $4 \mathrm{H}), 2.40-2.33(\mathrm{~m}, 2 \mathrm{H}), 2.23-2.20(\mathrm{~m}, 1 \mathrm{H}), 1.79-1.77$ (brs, $4 \mathrm{H}), 1.63-1.58(\mathrm{~m}, 2 \mathrm{H}), 1.27$ (brs, $2 \mathrm{H}) ;{ }^{13} \mathrm{C}$ NMR $\left(400 \mathrm{MHz}, \mathrm{CDCl}_{3}\right) \delta(\mathrm{ppm}) 166.58,161.72,143.95,134.30,133.36$ (q, J = 3.6 Hz), 129.60 (q, J =3 $5.9 \mathrm{~Hz}$ ), 126.75, 126.00(q, J = 3.6 Hz), 122.42 (q, J = 272.9 Hz), 64.37, 63.13, 51.99, 46.78,34.48, 33.80, 30.84, 27.24; ESI-MS (m/z): $515.6(\mathrm{M}+\mathrm{H})^{+}$.

\subsection{MIC determination}

MICs against replicating M. tuberculosis were determined by the microplate Alamar blue assay (MABA). RIF and INH were included as positive controls. M. tuberculosis $\mathrm{H} 37 \mathrm{Rv}$ and clinical isolate strains was grown to late log phase (70 to 100 Klett units) in Difco Middlebrook 7H9 Broth (catalog no. 271310) supplemented with 0.2\% (vol/vol) glycerol, $0.05 \%$ Tween 80, and 10\% (vol/vol) albumin-dextrosecatalase (BBL Middlebrook ADC Enrichment, catalog no. 212352) (7H9-ADCTG). Cultures were centrifuged, washed twice, and then suspended in phosphate phosphate-buffered saline. Suspensions were then passed through an $8 \mu \mathrm{m}$-pore-size filter to remove clumps, and aliquots were frozen at $-80^{\circ} \mathrm{C}$. Two fold dilutions of target compounds were prepared in 7H9-ADC-TG in a volume of $100 \mu \mathrm{l}$ in 96-well, black, clear-bottom microplates (BD Biosciences, Franklin Lakes, NJ). M. tuberculosis (100 $\mu \mathrm{l}$ containing $2 \times 10^{5} \mathrm{CFU}$ ) was added, yielding a final testing volume of $200 \mu 1$. The plates were incubated at $37^{\circ} \mathrm{C}$; on day 7 of incubation, $12.5 \mu 1$ of $20 \%$ Tween 80 and $20 \mu 1$ of Alamar blue were added to all wells. After incubation at $37^{\circ} \mathrm{C}$ for 16 to $24 \mathrm{~h}$, the fluorescence was read at an excitation of $530 \mathrm{~nm}$ and an emission of $590 \mathrm{~nm}$. The MIC was defined as the lowest concentration effecting a reduction in fluorescence of $\geq 90 \%$ relative to the mean of replicate bacterium-only controls.

\subsection{Pharmacokinetic Profiles determination}

SPF female ICR mice weighing 20-25 g were used in the pharmacokinetic study. The rats were fasted overnight before dosing. Every treatment group contained 3 mice. Mice were dosed with the tested compounds suspension at $50 \mathrm{mg} / \mathrm{kg}$ (p.o.). Compounds were suspended in $0.5 \% \mathrm{CMC}$ for oral administration. Blood was collected from the jugular vein of each animal at the following times after administration of drugs: $0.25,0.5,1,2,4,6,8$ and $24 \mathrm{~h}$ after a single oral dosing. All blood samples were centrifuged at $3000 \mathrm{r} / \mathrm{min}$ for $10 \mathrm{~min}$ to obtain serum which was then stored at $-20^{\circ} \mathrm{C} .150 \mu \mathrm{L}$ of the serum was added to $500 \mu \mathrm{L}$ of acetonitrile and the mixture was centrifuged at $13000 \mathrm{r} / \mathrm{min}$ for $10 \mathrm{~min}$ to remove protein. The supernatant was dried and dissolved in $100 \mu \mathrm{L}$ of acetonitrile, the solution was centrifuged at $13000 \mathrm{r} / \mathrm{min}$ for $10 \mathrm{~min}$. The supernatant was moved to a sample bottle for HPLC analysis. Total area under the concentration time curve (AUC), the elimination half-time $\left(t_{1 / 2}\right)$, the peak concentration $\left(C_{\max }\right)$ and the time to reach peak concentration $\left(\mathrm{T}_{\max }\right)$ of samples were determined directly from the experimental data using WinNonlin V6.2.1.

4.5 HPLC purity determination.

All samples were performed on an Agilent 1260 HPLC-UV system. Conditions (solvent $\mathrm{A}=$ methanol, solvent $\mathrm{B}=$ $\left.0.1 \% \mathrm{TFA}+\mathrm{H}_{2} \mathrm{O}\right)$ : Zorbax SB-C18 column $(250 \mathrm{~mm} \times 4.6 \mathrm{~mm}, 5 \mu \mathrm{m}$, PN: 883975-902). Injection volumn: $10 \mu \mathrm{L}$. Flow: $1.3 \mathrm{~mL} / \mathrm{min}$. Gradient elution: $0.00 \mathrm{~min}, 10 \% \mathrm{~A} ; 3 \mathrm{~min}, 50 \% \mathrm{~A} ; 15 \mathrm{~min}, 100 \% \mathrm{~A} ; 16 \mathrm{~min}, 10 \% \mathrm{~A} ; 18 \mathrm{~min} 10 \% \mathrm{~A}$. $\mathrm{UV}$ at $254 \mathrm{~nm}$.

\section{Notes}

The authors declare no competing financial interest.

\section{Abbreviations}


TB, tuberculosis; MTB, Mycobacterium tuberculosis; MDR, multidrug-resistant; XDR, extensively drug-resistant; WHO, World Health Organization; ATP, adenosine triphosphate; hERG, human ether-a-go-go related gene; ADME, absorption, distribution, metabolism, excretion, toxicity; BTZs, nitrobenzothiazinones; DprE1, Decaprenyl phosphoryl- $\beta$-D-ribose 2/-epimerase; SAR, structure-activity relationship; $\mathrm{PK}$, pharmacokinetic; $\mathrm{MeOH}$, methanol; $\mathrm{NaCNBH} 3$, sodium cyanoborohydride; TFA, trifluoroacetic acid; DCM, methylene chloride; MIC, minimum inhibitory concentration; HOBt, hydroxybenzotriazole; EDC, 1-ethyl-3-(3-dimethylaminopropyl)carbodiimide; LiAlH4, lithium aluminum hydride; MABA, microplate alamar blue assay; INH, isoniazid; RFP, rifampicin; T1/2, half-life; Cmax, the maximum serum concentration; Tmax, the time at which the Cmax is observed; AUC, area under the curve; MRT, mean residence time.

\section{Ethical statement}

All animal experiments were carried out in accordance with the guidelines of the Chinese Association for Laboratory Animal Sciences, and approved by the institutional ethical committee (IEC) of Peking Union Medical College.

\section{Acknowledgment}

This work is supported by the National Mega-project for Innovative Drugs (2017ZX09201001; 2015ZX09102007-008; 2015ZX09304006-016), CAMS Initiative for Innovative Medicine (2016-I2M-1-010), CAMS Innovation Fund for Medical Science (CAMS-2017-I2M-1-011), PUMC Youth Fund (2017350011) and Beijing Municipal Administration of Hospitals Clinical Medicine Development of Special Funding Support (ZYLX201304).

\section{References}

[1] A. Nusrath Unissa, L.E. Hanna, S. Swaminathan, A note on derivatives of isoniazid, rifampicin, and pyrazinamide showing activity against resistant mycobacterium tuberculosis, Chemical biology \& drug design, 87 (2016) 537-550. [2] Global tuberculosis report 2017. World Health Organization, http://www.who.int/tb/publications/global_report/en/.

[3] X. Li, V. Hernandez, F.L. Rock, W. Choi, Y.S.L. Mak, M. Mohan, W. Mao, Y. Zhou, E.E. Easom, J.J. Plattner, W. Zou, E. Perez-Herran, I. Giordano, A. Mendoza-Losana, C. Alemparte, J. Rullas, I. Angulo-Barturen, S. Crouch, F. Ortega, D. Barros, M.R.K. Alley, Discovery of a potent and specific M. tuberculosis leucyl-tRNA synthetase inhibitor: (S)-3-(Aminomethyl)-4-chloro-7-(2-hydroxyethoxy)benzo[c][1,2] oxaborol-1(3H)-ol (GSK656), J. Med. Chem. 60 (2017) 8011-8026.

[4] J. Cohen, Infectious disease. Approval of novel TB drug celebrated--with restraint, Science, 339 (2013) 130.

[5] N.J. Ryan, J.H. Lo, Delamanid: first global approval, Drugs, 74 (2014) 1041-1045.

[6] D.T. Hoagland, J. Liu, R.B. Lee, R.E. Lee, New agents for the treatment of drug-resistant Mycobacterium tuberculosis, Adv. Drug Deliv. Rev. 102 (2016) 55-72.

[7] V. Makarov, G. Manina, K. Mikusova, U. Mollmann, O. Ryabova, B. Saint-Joanis, N. Dhar, M.R. Pasca, S. Buroni, A.P. Lucarelli, A. Milano, E. De Rossi, M. Belanova, A. Bobovska, P. Dianiskova, J. Kordulakova, C. Sala, E. Fullam, P. Schneider, J.D. McKinney, P. Brodin, T. Christophe, S. Waddell, P. Butcher, J. Albrethsen, I. Rosenkrands, R. Brosch, V. Nandi, S. Bharath, S. Gaonkar, R.K. Shandil, V. Balasubramanian, T. Balganesh, S. Tyagi, J. Grosset, G. Riccardi, S.T. Cole, Benzothiazinones kill Mycobacterium tuberculosis by blocking arabinan synthesis, Science, 324 (2009) 801-804. 
[8] V. Makarov, B. Lechartier, M. Zhang, J. Neres, A.M. van der Sar, S.A. Raadsen, R.C. Hartkoorn, O.B. Ryabova, A. Vocat, L.A. Decosterd, N. Widmer, T. Buclin, W. Bitter, K. Andries, F. Pojer, P.J. Dyson, S.T. Cole, Towards a new combination therapy for tuberculosis with next generation benzothiazinones, EMBO Mol. Med. 6 (2014) 372-383.

[9] C. Trefzer, M. Rengifo-Gonzalez, M.J. Hinner, P. Schneider, V. Makarov, S.T. Cole, K. Johnsson, Benzothiazinones: prodrugs that covalently modify the decaprenylphosphoryl-beta-D-ribose 2'-epimerase DprE1 of Mycobacterium tuberculosis, J. Am. Chem. Soc. 132 (2010) 13663-13665.

[10] T. Karoli, B. Becker, J. Zuegg, U. Mollmann, S. Ramu, J.X. Huang, M.A. Cooper, Identification of antitubercular benzothiazinone compounds by ligand-based design, J. Med. Chem. 55 (2012) 7940-7944.

[11] K. Lv, X. You, B. Wang, Z. Wei, Y. Chai, B. Wang, A. Wang, G. Huang, M. Liu, Y. Lu, Identification of better pharmacokinetic benzothiazinone derivatives as new antitubercular agents, ACS Med. Chem. Lett. 8 (2017) 636-641.

[12] R. Zhang, K. Lv, B. Wang, L. Li, B. Wang, M. Liu, H. Guo, A. Wang, Y. Lu, Design, synthesis and antitubercular evaluation of benzothiazinones containing an oximido or amino nitrogen heterocycle moiety, Rsc Adv. 7 (2017) $1480-1483$.

[13] J. Neres, F. Pojer, E. Molteni, L.R. Chiarelli, N. Dhar, S. Boy-Rottger, S. Buroni, E. Fullam, G. Degiacomi, A.P. Lucarelli, R.J. Read, G. Zanoni, D.E. Edmondson, E. De Rossi, M.R. Pasca, J.D. McKinney, P.J. Dyson, G. Riccardi, A. Mattevi, S.T. Cole, C. Binda, Structural basis for benzothiazinone-mediated killing of mycobacterium tuberculosis, Sci. Transl. Med. 4 (2012) 150ra121.

[14] F. Kloss, V. Krchnak, A. Krchnakova, S. Schieferdecker, J. Dreisbach, V. Krone, U. Mollmann, M. Hoelscher, M.J. Miller, In vivo dearomatization of the potent antituberculosis agent BTZ043 via Meisenheimer Complex Formation, Angew Chem. Int. Edit. 56 (2017) 2187-2191.

[15] R. Tiwari, P.A. Miller, L.R. Chiarelli, G. Mori, M. Sarkan, I. Centarova, S.H. Cho, K. Mikusova, S.G. Franzblau, A.G. Oliver, M.J. Miller, Design, Syntheses, and Anti-TB Activity of 1,3-Benzothiazinone Azide and Click Chemistry Products Inspired by BTZ043, ACS Med. Chem. Lett. 7 (2016) 266-270.

[16] V. Makarov, J. Neres, R.C. Hartkoorn, O.B. Ryabova, E. Kazakova, M. Sarkan, S. Huszar, J. Piton, G.S. Kolly, A. Vocat, T.M. Conroy, K. Mikusova, S.T. Cole, The 8-Pyrrole-Benzothiazinones Are Noncovalent Inhibitors of DprE1 from Mycobacterium tuberculosis, Antimicrobial agents and chemotherapy, 59 (2015) 4446-4452.

[17] C.T. Peng, C. Gao, N.Y. Wang, X.Y. You, L.D. Zhang, Y.X. Zhu, Y. Xv, W.Q. Zuo, K. Ran, H.X. Deng, Q. Lei, K.J. Xiao, L.T. Yu, Synthesis and antitubercular evaluation of 4-carbonyl piperazine substituted 1,3-benzothiazin-4-one derivatives, Bioorganic \& medicinal chemistry letters, 25 (2015) 1373-1376.

[18] R. Tiwari, U. Mollmann, S. Cho, S.G. Franzblau, P.A. Miller, M.J. Miller, Design and Syntheses of Anti-Tuberculosis Agents Inspired by BTZ043 Using a Scaffold Simplification Strategy, ACS medicinal chemistry letters, 5 (2014) 587-591.

[19] C. Gao, T.H. Ye, N.Y. Wang, X.X. Zeng, L.D. Zhang, Y. Xiong, X.Y. You, Y. Xia, Y. Xu, C.T. Peng, W.Q. Zuo, Y. Wei, L.T. Yu, Synthesis and structure-activity relationships evaluation of benzothiazinone derivatives as potential anti-tubercular agents, Bioorganic \& medicinal chemistry letters, 23 (2013) 4919-4922.

[20] R. Tiwari, P.A. Miller, S. Cho, S.G. Franzblau, M.J. Miller, Syntheses and Antituberculosis Activity of 1,3-Benzothiazinone Sulfoxide and Sulfone Derived from BTZ043, ACS medicinal chemistry letters, 6 (2015) 128-133.

[21] Y. Lu, M. Zheng, B. Wang, L. Fu, W. Zhao, P. Li, J. Xu, H. Zhu, H. Jin, D. Yin, H. Huang, A.M. Upton, Z. Ma, Clofazimine analogs with efficacy against experimental tuberculosis and reduced potential for accumulation, Antimicrob. agents Chemother. 55 (2011) 5185-5193. 
Table 1. Structures and activities of $\mathbf{1 a - b}$ and $\mathbf{2 a - e}$

20

INH: isoniazid; RFP: rifampicin 
Table 2. Structures and activities of 1a-b and 2a-e<smiles>N#CC1CCN(c2nc(=O)c3cc(C(F)(F)F)cc([N+](=O)[O-])c3s2)CC1</smiles>

\begin{tabular}{|c|c|c|}
\hline Compd. & W & MIC \\
\hline $2 a$ & & 0.481 \\
\hline $2 f$ & & 1.922 \\
\hline $2 \mathrm{~g}$ & & 0.241 \\
\hline $2 \mathrm{~h}$ & & 0.488 \\
\hline $2 \mathbf{i}$ & & $<0.016$ \\
\hline $2 \mathbf{j}$ & & 1.961 \\
\hline $2 k$ & & 3.709 \\
\hline PBTZ1 & & $<0.016$ \\
\hline
\end{tabular}


Table 3. Anti-MDR TB activity of compound $\mathbf{2 i}$

\begin{tabular}{lll}
\hline \multirow{2}{*}{ Compd. } & \multicolumn{2}{c}{ MIC $(\mu \mathrm{g} / \mathrm{mL})$} \\
\cline { 2 - 3 } & MDR-TB $16995^{\mathrm{a}}$ & MDR-TB 16883 \\
\hline $\mathbf{2 i}$ & $<0.016$ & $<0.016$ \\
PBTZ169 & $<0.016$ & $<0.016$ \\
INH & $>40$ & $>40$ \\
RFP & $>40$ & $>40$
\end{tabular}

INH: isoniazid; RFP: rifampicin; ${ }^{\mathrm{a}}$ MDR-TB 16995 and MDR-TB 16883 were isolated from patients in Beijing Chest Hospital.

Table 4. PK profiles of compound $2 \mathbf{i}$ dosed orally in mice at $50 \mathrm{mg} / \mathrm{kg}(\mathrm{n}=3)$

\begin{tabular}{lll}
\hline PK parameters & $\mathbf{2 i}$ & PBTZ169 \\
\hline $\mathrm{T}_{1 / 2}(\mathrm{~h})$ & $3.3 \pm 3.01$ & $2.87 \pm 1.03$ \\
Tmax (h) & $0.25 \pm 0$ & $0.83 \pm 0.29$ \\
Cmax $(\mathrm{ng} / \mathrm{mL})$ & $1165 \pm 223$ & $1300 \pm 422$ \\
AUC $_{0-\text { inf }}(\mathrm{h} \cdot \mathrm{ng} / \mathrm{mL})$ & $2489 \pm 1273$ & $5478 \pm 1730$ \\
MRT $(\mathrm{h})$ & $4.26 \pm 3.74$ & $3.73 \pm 0.94$ \\
\hline
\end{tabular}




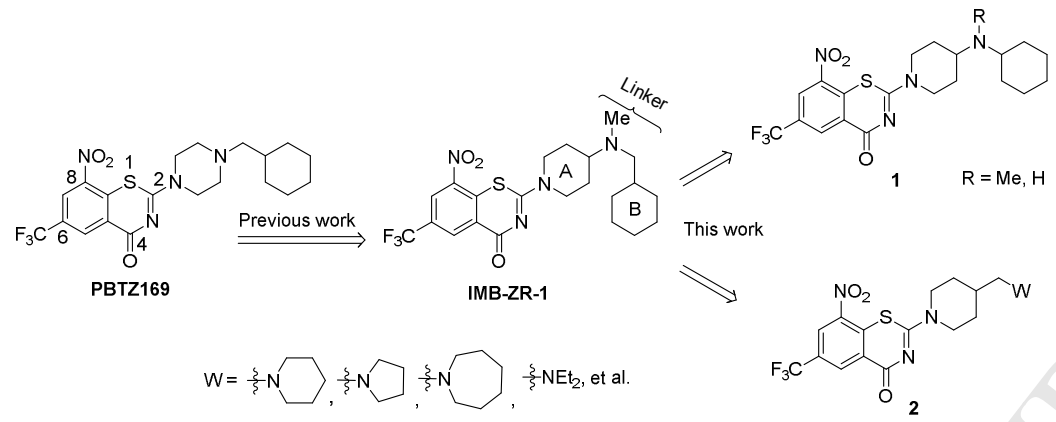

Figure 1. Design of new BTZs

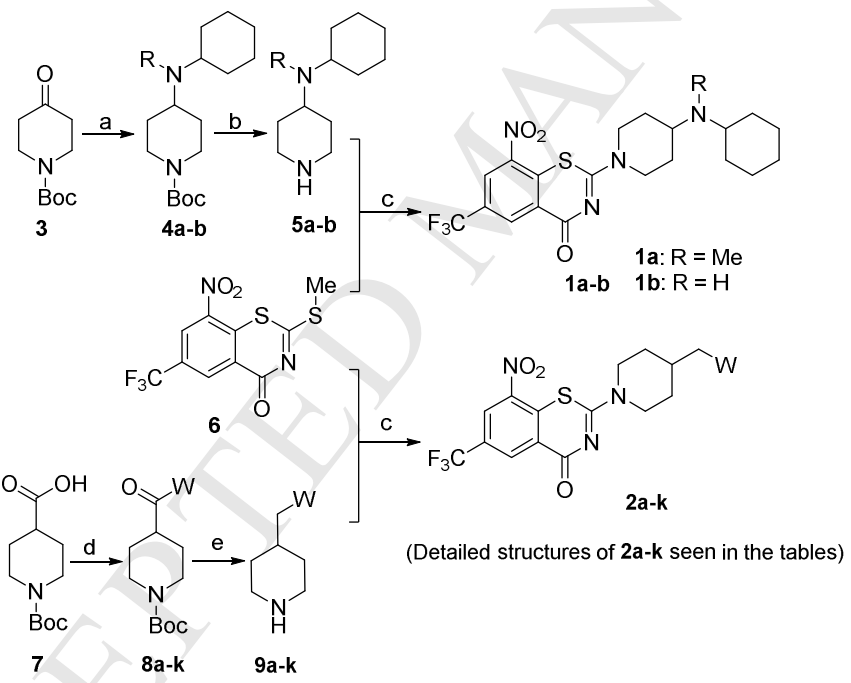

a) $\mathrm{NaCNBH}_{3}, \mathrm{MeOH}$, cyclohexylamine or $\mathrm{N}$-methylcyclohexylamine, $\mathrm{AcOH}, \mathrm{rt}, 2 \mathrm{hrs}$; b) TFA, DCM, $0^{\circ} \mathrm{C}, 0.5 \mathrm{~h}$; c) Et $3 \mathrm{~N}, \mathrm{MeOH}, \mathrm{rt}, 3 \mathrm{hrs}$; d) EDC, HOBt, Et $3 \mathrm{~N}, \mathrm{DCM}, \mathrm{rt}, 5$ hrs; e) i: TFA, DCM, $0^{\circ} \mathrm{C}, 0.5 \mathrm{~h}$, ii: $1.0 \mathrm{M} \mathrm{LiAlH}_{4}$ in THF, THF, $0^{\circ} \mathrm{C}, 1 \mathrm{~h}$.

Scheme 1. Synthesis of BTZs 1a-b and 2a-k 


\section{Highlights:}

1. Two series of BTZs 1-2 with piperidine moiety were designed and synthesized.

2. Some targets showed considerable in vitro activity (MIC $<1 \mu \mathrm{g} / \mathrm{mL}$ ) against MTB strain.

3. Compound $2 \mathbf{i}$ displayed potent in vitro anti-MDR-TB activity (MIC $<0.016 \mu \mathrm{g} / \mathrm{mL}$ ). 